\title{
OPEN Comparison of corneal irregular astigmatism by the type of corneal regular astigmatism
}

\author{
Yuta Ueno ${ }^{1}$, Risa Nomura ${ }^{1}$, Takahiro Hiraoka ${ }^{1}$, Katsuhito Kinoshita ${ }^{1}$, Mutsuko Ohara ${ }^{2} \&$ \\ Tetsuro Oshika ${ }^{1 凶}$
}

We investigated the relation between corneal regular and irregular astigmatism in normal human eyes. In 951 eyes of 951 patients, corneal irregular astigmatism, such as asymmetry and higher-order irregularity components, was calculated using the Fourier harmonic analysis of corneal topography data within the central 3-mm zone of the anterior corneal surface. The eyes were classified by the type of corneal regular astigmatism into four groups; minimum ( $<0.75$ diopters), with-the-rule (WTR), against-the-rule (ATR), and oblique astigmatism. The mean age was significantly different among the four groups $(P<0.001)$; patients with WTR astigmatism were the youngest, followed by those with minimum, oblique, and ATR astigmatism. Significant inter-group differences were found among the four groups in asymmetry $(P=0.005)$ and higher-order irregularity components $(P<0.001)$; the largest was in eyes with oblique astigmatism, followed by ATR, WTR, and minimum astigmatism. The stepwise multiple regression analysis revealed that corneal regular astigmatism pattern significantly influenced the amount of corneal irregular astigmatism after controlling for confounding factors $(P<0.001)$. Corneal irregular astigmatism, such as asymmetry and higher order irregularity components, was the largest in eyes with oblique astigmatism, followed by those with ATR, WTR, and minimum astigmatism, even after adjustment for age of subjects.

The cornea has two forms of astigmatism; regular and irregular astigmatism. Among them, irregular astigmatism is defined as astigmatism that cannot be corrected with spherocylindrical spectacle lenses, and thus is one of the important causes of suboptimal visual function, such as corrected visual acuity and contrast sensitivity, in otherwise healthy eyes ${ }^{1,2}$.

Pathological changes in corneal irregular astigmatism can be induced by various kind of corneal diseases ${ }^{3-8}$ as well as ocular surgeries ${ }^{9-14}$. Physiologically, corneal irregular astigmatism in normal human eyes is known to increase along with aging ${ }^{15-21}$. The relation between corneal regular and irregular astigmatism, however, has not been studied in detail, and it is yet to be clarified whether there is any association between corneal irregular astigmatism and the type of corneal regular astigmatism, such as with-the-rule (WTR), against-the-rule (ATR), and oblique astigmatism. Previous studies ${ }^{22-24}$ have shown that eyes with oblique astigmatism showed worse visual functions than those with ATR astigmatism, and that eyes with WTR astigmatism were least affected. After monofocal intraocular lens implantation, uncorrected distance visual acuity (UDVA) in eyes with simple myopic ATR was worse than in eyes with simple myopic WTR astigmatism ${ }^{25}$. It is, therefore, of clinical as well as pathophysiological interest to assess corneal irregular astigmatism in normal human eyes in relation to the pattern of corneal regular astigmatism.

\section{Patients and methods}

Patients. We retrospectively collected the data of patients who had undergone ocular examinations at Tsukuba University Hospital or Ito Eye Clinic. Patients were selected from the consecutive cohort according to the following criteria. Eyes were excluded from the study if they had ocular diseases except for mild to moderate cataract, any history of eye surgery including cataract surgery, or use of a contact lens within 3 weeks. Eyes with advanced cataract were excluded due to possible poor visual fixation during measurements.

Examinations. The eyes were examined with swept-source anterior segment optical coherence tomography (AS-OCT) (CASIA, SS-1000, Tomey, Nagoya, Japan), which is a three-dimensional Fourier domain OCT using 


\begin{tabular}{|l|l|l|l|l|}
\hline \multirow{2}{*}{} & \multicolumn{4}{|l|}{ Type of corneal regular astigmatism } \\
\cline { 2 - 5 } & Minimum (<0.75 D) & WTR & ATR & Oblique \\
\hline Number of eyes & 350 & 334 & 194 & 73 \\
\hline Male/female & $157 / 193$ & $161 / 173$ & $99 / 95$ & $32 / 41$ \\
\hline Age (years old) & $65.9 \pm 15.1(7-88)$ & $47.0 \pm 22.0(6-90)$ & $73.8 \pm 8.5(41-93)$ & $66.4 \pm 17.4(8-89)$ \\
\hline
\end{tabular}

Table 1. Background data of patients. D: diopter, $W T R$ with-the-rule, ATR against-the-rule, mean \pm standard deviation (range).

a 1,310-nm light source. Its axial and transverse resolutions are $10 \mu \mathrm{m}$ and $30 \mu \mathrm{m}$, respectively. The device uses auto alignment and auto focus of the examined eyes. The scanning speed is 30,000 A-scans per second, and the acquisition time is $\leq 0.3 \mathrm{~s}$. Its accuracy and reliability of corneal measurements have been reported elsewhere $\mathrm{e}^{26,27}$. The quality of AS-OCT images was confirmed by the examiner after each measurement before storage.

From the anterior corneal curvature data, regular astigmatism was calculated using the keratometric index. Based on corneal regular astigmatism, the eyes were classified into four groups; minimum astigmatism $(<0.75$ diopters, D), WTR astigmatism, ATR astigmatism, and oblique astigmatism groups. When the steepest meridian of the anterior cornea was within \pm 30 degrees of the vertical axis, the astigmatism was judged to be WTR. Those with the steepest meridian of \pm 30 degrees of the horizontal axis were considered to have ATR astigmatism. All others were regarded as oblique astigmatism.

For the calculation of irregular astigmatism, anterior corneal dioptric data of AS-OCT were expanded into spherical power, asymmetry component (first order harmonic), regular astigmatism (second order harmonic), and higher-order irregularity component (third and higher order harmonic) using Fourier harmonic analysis ${ }^{28}$. Asymmetry and higher-order irregularity components computed within the central 3-mm zone were used to represent corneal irregular astigmatism.

The study protocol was reviewed and approved by the Institutional Review Board of University of Tsukuba. This study was conducted in accordance with the Declaration of Helsinki. The informed consent was obtained from each patient.

Statistical analysis. For assessment of data among four groups, the multiple comparison test was employed, i.e. one-way analysis of variance (ANOVA) with Bonferroni correction. The stepwise multiple regression analysis was performed to evaluate the influence of age and type of regular astigmatism (explanatory variables) on the amount of irregular astigmatism (dependent variable). The type of regular astigmatism was converted to dummy variables, such as $(1,0,0,0),(0,1,0,0),(0,0,1,0)$, and $(0,0,0,1)$. All statistical tests were 2 -sided and a $p$-value of less than 0.05 was considered significant. The numerical data are presented as the mean \pm standard deviation (SD) unless otherwise noted. Statistical analysis was performed using SPSS Statistics for Windows software (version 26, IBM Corp., Armonk, NY, USA).

Results. Data from 951 eyes of 951 patients were collected and analysed. There were 449 males and 502 females, and their age averaged $60.9 \pm 20.1$ (range 6-93) years old. The background data of patients are shown in Table 1. There was a significant difference in the mean age among groups $(P<0.001)$, and the post-hoc test revealed significant differences between each group except for between minimum and oblique astigmatism groups (Fig. 1). The patients with WTR astigmatism were the youngest, followed by those with minimum, oblique, and ATR astigmatism.

Significant differences were found among the four groups in the amount of corneal irregular astigmatism, such as asymmetry $(P=0.005)$ and higher-order irregularity components $(P<0.001)$. The asymmetry component was significantly smaller in the minimum astigmatism group than in the oblique astigmatism groups $(P=0.050)$, while statistically significant differences were not found between other groups (Fig. 2). In the higher-order irregularity component, significant inter-group differences were found for every match-up (Fig. 3), except for between the minimum and WTR astigmatism groups $(P=1.000)$.

In all 951 eyes, the amount of regular astigmatism significantly correlated with those of asymmetry $(r=0.092$, $P=0.004)$ and higher-order irregularity $(\mathrm{r}=0.164, P<0.004)$. In eyes with minimum astigmatism, regular astigmatism did not correlate with asymmetry $(\mathrm{r}=-0.015, P=0.402)$ and higher-order irregularity $(\mathrm{r}=0.075$, $P=0.160)$. In eyes with WTR astigmatism, regular astigmatism significantly correlated with asymmetry $(\mathrm{r}=0.155$, $P=0.004)$ and higher-order irregularity $(\mathrm{r}=0.162, P=0.003)$. In eyes with ATR astigmatism, regular astigmatism did not correlate with asymmetry $(r=-0.028, P=0.694)$ and higher-order irregularity $(r=0.0782 P=0.225)$. In eyes with oblique astigmatism, regular astigmatism did not correlate with asymmetry $(\mathrm{r}=0.055, P=0.643)$, but significantly correlated with higher-order irregularity $(\mathrm{r}=0.534, P<0.001)$.

The stepwise multiple regression analysis revealed that age (partial correlation coefficient $r=0.258, P<0.001$ ) and type of regular astigmatism (WTR astigmatism $\mathrm{r}=0.157, P<0.001$, oblique astigmatism $\mathrm{r}=0.082, P=0.011$ ) were significantly associated with the asymmetry component. Parameters that were significantly relevant to the higher-order irregularity component included age $(\mathrm{r}=0.161, P<0.001)$ and type of regular astigmatism (minimum astigmatism $r=-0.097, P=0.003$, oblique astigmatism $r=0.129, P<0.001$ ). 


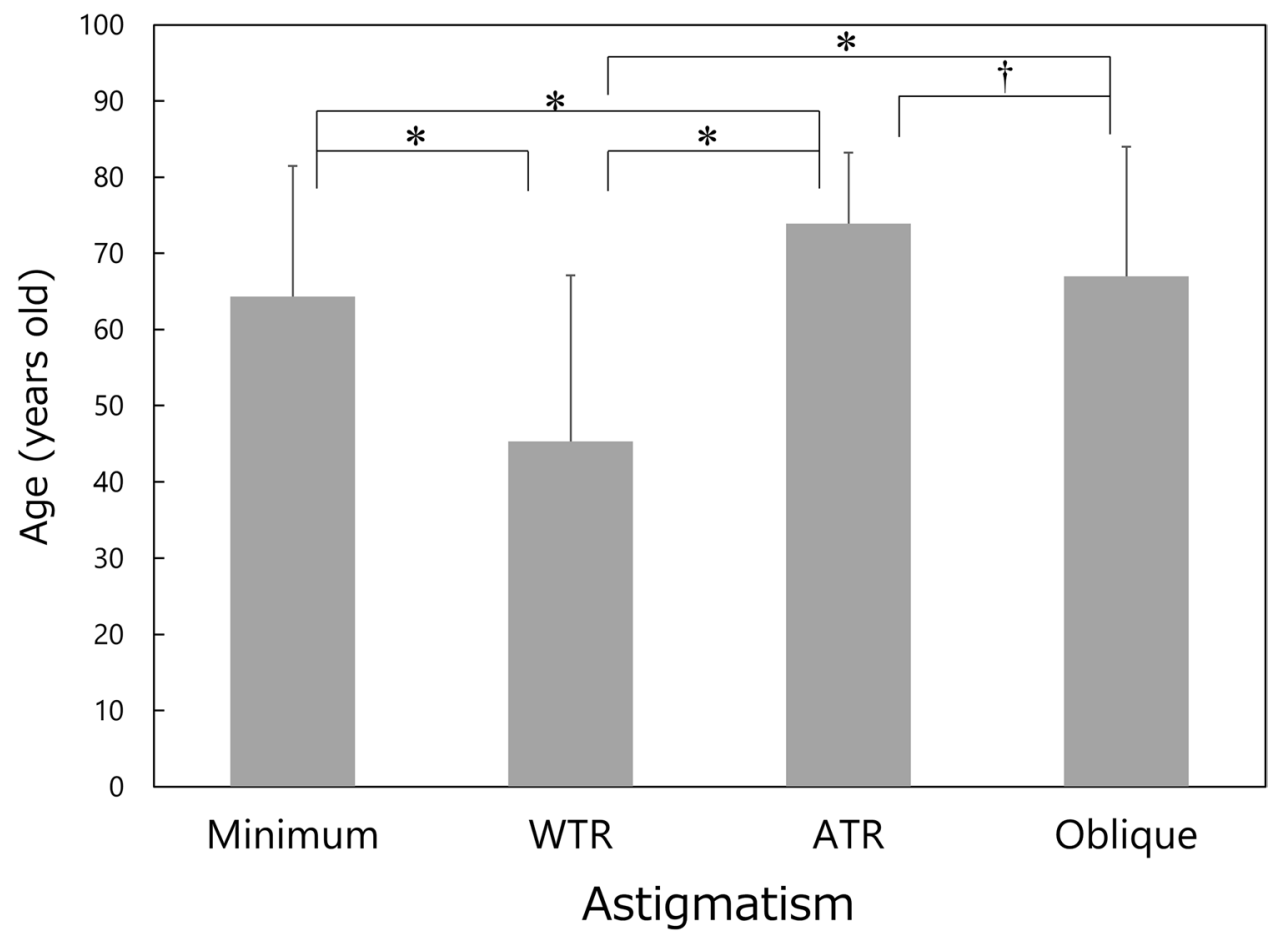

Figure 1. Comparison of age among the four types of corneal regular astigmatism groups. Mean \pm standard deviation, $W T R$ with-the-rule, $A T R$ against-the-rule, ${ }^{\star} P<0.001, \dagger P=0.010$.

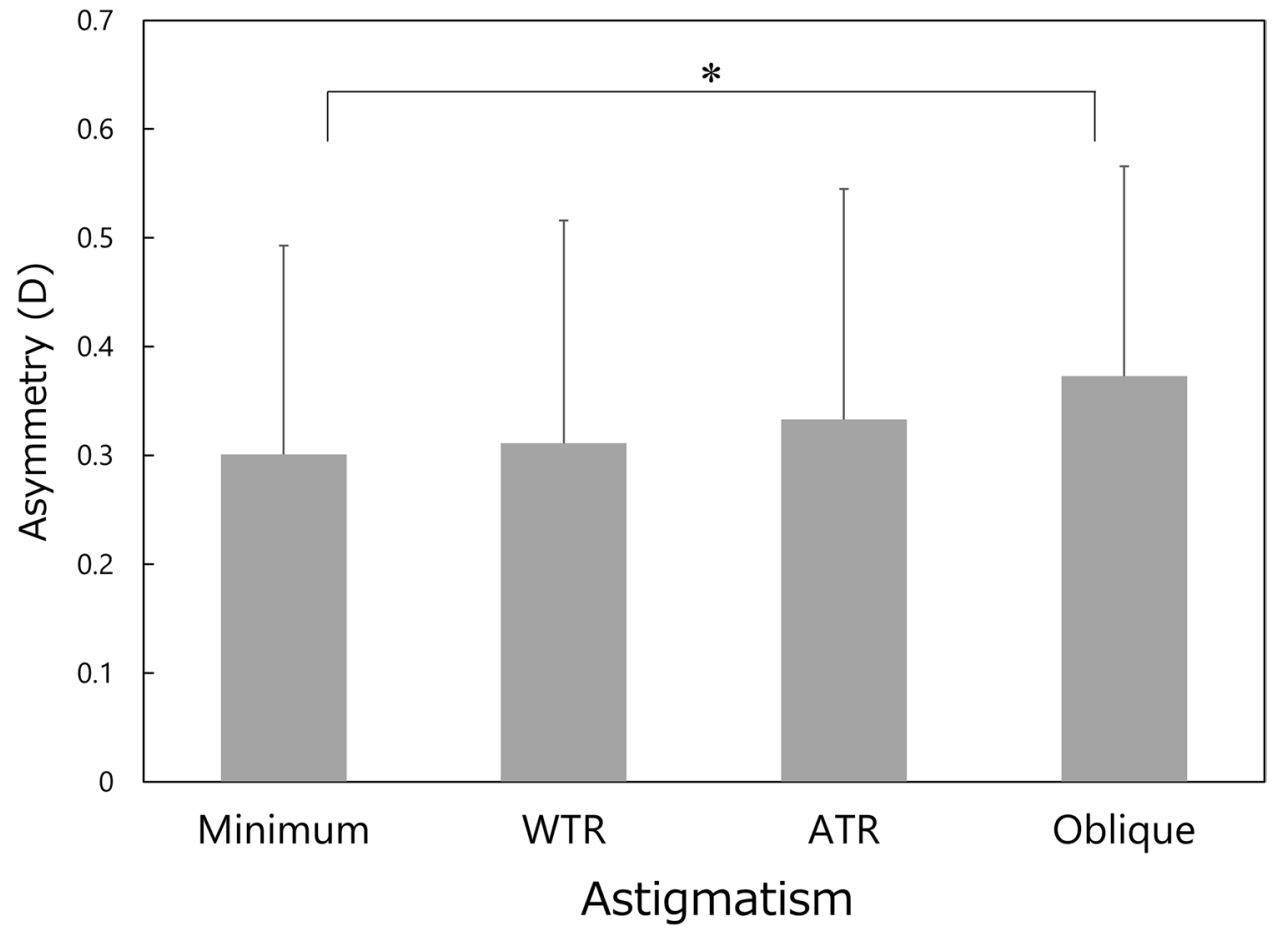

Figure 2. Comparison of asymmetry component among the four types of corneal regular astigmatism groups. Mean \pm standard deviation, $W T R$ with-the-rule, ATR against-the-rule, ${ }^{*} P=0.050$. 


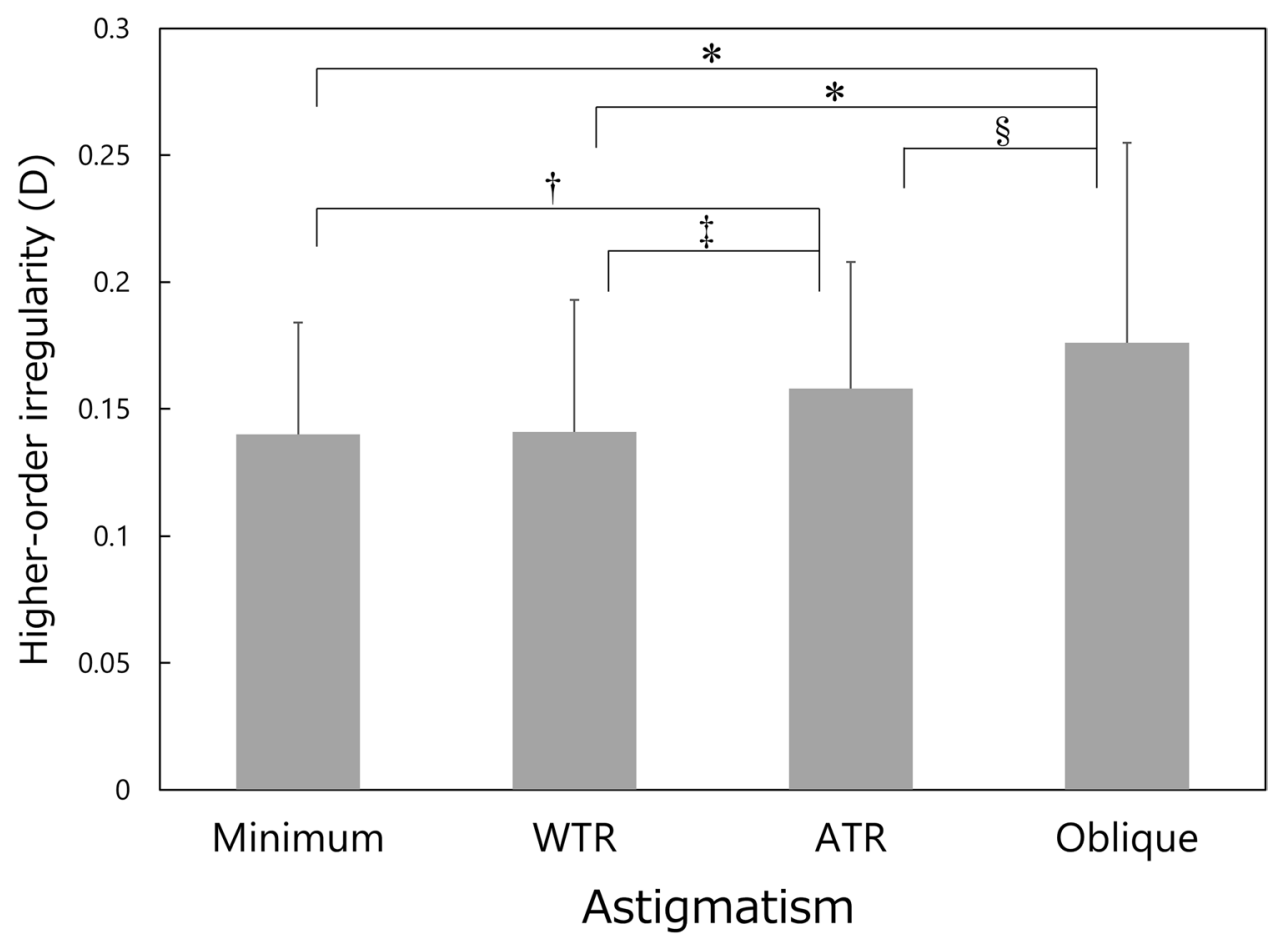

Figure 3. Comparison of higher-order irregularity component among the four types of corneal regular astigmatism groups. Mean \pm standard deviation, $W T R$ with-the-rule, ATR against-the-rule, ${ }^{\star} P<0.001$, $\dagger P=0.009, \ddagger P=0.044, \S P=0.015$.

\section{Discussion}

In general, corneal irregular astigmatism, including higher-order aberrations, increases along with aging in normal human eyes ${ }^{15-21}$. In the current study, the multiple regression analysis indicated that age is one of the important factors that contribute to the increases of corneal irregular astigmatism. When comparing the age of each group (Fig. 1), subjects in the ATR astigmatism group were the oldest, followed by those in the oblique, minimum, and WTR astigmatism groups. This pattern (order of four groups) is different from that of corneal irregular astigmatism, including asymmetry (Fig. 2) and higher-order irregularity component (Fig. 3), in which the parameters were the largest in eyes with oblique astigmatism, followed by those with ATR, WTR, and minimum astigmatism. In addition, the stepwise multiple regression revealed that the type of corneal regular astigmatism significantly contributes to the degree of corneal irregular astigmatism, after adjustment for age. These results indicate that corneal irregular astigmatism is influenced by the type of corneal regular astigmatism, independently of the age of subjects.

To the best of our knowledge, the current study represents the first report on the association between corneal irregular astigmatism and the type of corneal regular astigmatism, by controlling for confounding factors. In a previous study, Kiuchi et al. ${ }^{29}$ evaluated 426 patients with AS-OCT and reported that age and the amount of corneal regular astigmatism showed significant correlations with corneal higher-order aberrations, whereas the amount of spherical equivalent and refractive status (myopia, emmetropia, or hyperopia) did not. Choi et al. ${ }^{30}$ investigated the changes in corneal higher-order aberration during amblyopia treatment, and assessed the correlation between higher-order aberration and astigmatism in hyperopic amblyopia children. They found that coma higher-order aberration significantly correlated with astigmatism and could exert effects in cases involving hyperopic amblyopia. Cheng et al. ${ }^{31}$ examined the relationship between ametropia and optical aberrations in a population of 200 normal human eyes with refractive errors spanning the range from +5.00 to $-10.00 \mathrm{D}$, and reported that astigmatic eyes tended to have larger total higher-order aberrations than nonastigmatic eyes. Leung et al. ${ }^{32}$ evaluated the corneal shapes and monochromatic aberrations in Chinese myopic adults with and without astigmatism, and demonstrated significant relationships between astigmatism, corneal shapes, and monochromatic aberrations. Compared with simple myopia, myopic astigmatism had more oblate nasal and temporal corneal shapes and showed significantly more negative $Y$ trefoil and more positive vertical coma. The asymmetry in corneal shape along the vertical principal meridian (inferior-superior) was significantly associated with the $\mathrm{Y}$ trefoil and vertical coma of the cornea, suggesting that this regional asymmetry in corneal shape may contribute to the ocular aberrations. Our study indicated that eyes with oblique astigmatism is associated with the greatest amount of asymmetry component as well as high-order irregularity component, in line with the findings of aforementioned studies.

With a greater amount of corneal irregular astigmatism, optical quality of eyes with oblique astigmatism may be deteriorated to some extent. The influence of axis orientation of uncorrected astigmatism on visual function has been studied previously in normal eyes of healthy volunteers ${ }^{22-24}$ and eyes after monofocal intraocular lens 
implantation ${ }^{25}$. The studies in volunteers reported that oblique astigmatism induced with cylindrical lenses resulted in worse visual functions than ATR astigmatism, and that WTR astigmatism had the least effect ${ }^{22-24}$. In eyes after intraocular lens implantation, it was demonstrated that UDVA was worse in eyes with simple myopic ATR astigmatism than in those with simple myopic WTR astigmatism ${ }^{25}$. These studies, however, were about the influence of uncorrected corneal regular astigmatism on visual function. In order to elucidate the impact of naturally occurring corneal irregular astigmatism as in our study, the relation between spectacle-corrected visual functions and type of corneal regular astigmatism should be investigated, though such studies are not available until now. Further studies are awaited.

The present study has several limitations. First, being a retrospective study, some important data are missing, such as axial length and refractive status. Previous studies, however, showed that refractive status (myopia, emmetropia, or hyperopia) is not correlated with corneal higher-order aberrations when age is taken into account ${ }^{23,25}$, and thus we believe that our study is not largely biased by the absence of those data. Other factors, such as intraocular pressure, anterior chamber depth, corneal thickness, and corneal diameter were not analyzed in this study, which would be the subject of future studies. Second, we did not analyze the impact of corneal irregular astigmatism on visual function, such as visual acuity and contrast sensitivity. Thus, it is not clear to what extent the current findings have clinical relevance. Nonetheless, the current results indicate that eyes with oblique astigmatism are associated with deteriorated optical quality, where consideration is needed in the indication of toric intraocular lenses, selection of hard/soft contact lenses, and performance of surgical correction of astigmatism on the cornea. Third, we presented the data obtained from the measurements of corneal anterior surface alone, and the influence of posterior corneal astigmatism was not considered. Since the astigmatism pattern is totally different between the anterior and posterior corneal surfaces ${ }^{34-35}$, it seems more desirable to analyze the total corneal astigmatism by combining both anterior and posterior corneal astigmatism. These are the subjects of future studies.

In conclusion, we investigated the relation between corneal regular and irregular astigmatism. Corneal irregular astigmatism was calculated using Fourier harmonic analysis of corneal topography data obtained with ASOCT, and was analyzed according to the type of corneal regular astigmatism; minimum $(<0.75 \mathrm{D})$, WTR, ATR, and oblique astigmatism. It was found that corneal irregular astigmatism, such as asymmetry and higher order irregularity components, was the largest in eyes with oblique astigmatism, followed by those with ATR, WTR, and minimum astigmatism, even after adjustment for age of subjects.

Received: 4 June 2021; Accepted: 26 July 2021

Published online: 04 August 2021

\section{References}

1. Tomidokoro, A. et al. Corneal irregular astigmatism and contrast sensitivity after photorefractive keratectomy. Ophthalmology 108, 2209-2212 (2001).

2. Hiraoka, T., Okamoto, C., Ishii, Y., Okamoto, F. \& Oshika, T. Recovery of corneal irregular astigmatism, ocular higher-order aberrations, and contrast sensitivity after discontinuation of overnight orthokeratology. Br. J. Ophthalmol. 93, 203-208 (2009).

3. Tomidokoro, A., Oshika, T., Amano, S., Eguchi, K. \& Eguchi, S. Quantitative analysis of regular and irregular astigmatism induced by pterygium. Cornea 18, 412-415 (1999).

4. Oshika, T., Tanabe, T., Tomidokoro, A. \& Amano, S. Progression of keratoconus assessed by Fourier analysis of videokeratography data. Ophthalmology 109, 339-342 (2002).

5. Tanabe, T. et al. Corneal regular and irregular astigmatism assessed by Fourier analysis of videokeratography data in normal and pathologic eyes. Ophthalmology 111, 752-757 (2004).

6. Yoshihara, M. et al. Corneal topographic analysis of patients with Mooren ulcer using 3-dimensional anterior segment optical coherence tomography. Cornea 34, 54-59 (2015).

7. Koh, S. et al. Fourier analysis of corneal irregular astigmatism due to the anterior corneal surface in dry eye. Eye Contact Lens. 45, 188-194 (2019).

8. Oie, Y. et al. Fourier analysis on regular and irregular astigmatism of anterior and posterior corneal surfaces in Fuchs endothelial corneal dystrophy. Am. J. Ophthalmol. 223, 33-41 (2021).

9. Tomidokoro, A., Oshika, T. \& Kojima, T. Corneal astigmatism after scleral buckling surgery assessed by Fourier analysis of videokeratography data. Cornea 17, 517-521 (1998).

10. Hayashi, K., Hayashi, H., Oshika, T. \& Hayashi, F. Fourier analysis of irregular astigmatism after trabeculectomy. Ophthalmic Surg. Lasers. 31, 94-99 (2000).

11. Baek, T. M., Lee, K. H., Tomidokoro, A. \& Oshika, T. Corneal irregular astigmatism after laser in situ keratomileusis for myopia. Br. J. Ophthalmol. 85, 534-536 (2001).

12. Oshika, T., Sugita, G., Tanabe, T., Tomidokoro, A. \& Amano, S. Regular and irregular astigmatism after superior versus temporal scleral incision cataract surgery. Ophthalmology 107, 2049-2053 (2000).

13. Okamoto, F. et al. Changes in corneal topography after 25 -gauge transconjunctival sutureless vitrectomy versus after 20-gauge standard vitrectomy. Ophthalmology 114, 2138-2141 (2007).

14. Sideroudi, H., Sekundo, W., Kozobolis, V., Messerschmidt-Roth, A. \& Lazaridis, A. Fourier analysis of corneal irregular astigmatism after small incision lenticule extraction and comparison to femtosecond laser-assisted laser in situ keratomileusis. Cornea 38, 1536-1542 (2019).

15. Oshika, T., Klyce, S. D., Applegate, R. A. \& Howland, H. C. Changes in corneal wavefront aberrations with aging. Invest. Ophthalmol. Vis. Sci. 40, 1351-1355 (1999).

16. Artal, P., Berrio, E., Guirao, A. \& Piers, P. Contribution of the cornea and internal surfaces to the change of ocular aberrations with age. J. Opt. Soc. Am. A Opt. Image Sci. Vis. 19, 137-143 (2002).

17. Fujikado, T. et al. Age-related changes in ocular and corneal aberrations. Am. J. Ophthalmol. 138, 143-146 (2004).

18. Amano, S. et al. Age-related changes in corneal and ocular higher-order wavefront aberrations. Am. J. Ophthalmol. 137, 988-992 (2004).

19. KhabazKhoob, M. et al. Keratometry measurements, corneal astigmatism and irregularity in a normal population: the Tehran Eye Study. Ophthalmic Physiol. Opt. 30, 800-805 (2010).

20. Hayashi, K., Kawahara, S., Manabe, S. \& Hirata, A. Changes in irregular corneal astigmatism with age in eyes with and without cataract surgery. Invest. Ophthalmol. Vis. Sci. 56, 7988-7998 (2015). 
21. Kemraz, D. et al. Age-related changes in corneal spherical aberration. J. Refract. Surg. 34, 760-767 (2018).

22. Chen, S. I., Hove, M., McCloskey, C. L. \& Kaye, S. B. The effect of monocularly and binocularly induced astigmatic blur on depth discrimination is orientation dependent. Optom. Vis. Sci. 82, 101-113 (2005).

23. Wolffsohn, J. S., Bhogal, G. \& Shah, S. Effect of uncorrected astigmatism on vision. J. Cataract. Refract. Surg. 37, 454-460 (2011).

24. Kobashi, H., Kamiya, K., Shimizu, K., Kawamorita, T. \& Uozato, H. Effect of axis orientation on visual performance in astigmatic eyes. J. Cataract. Refract. Surg. 38, 1352-1359 (2012).

25. Yamamoto, T., Hiraoka, T., Beheregaray, S. \& Oshika, T. Influence of simple myopic against-the-rule and with-the-rule astigmatism on visual acuity in eyes with monofocal intraocular lenses. Jpn. J. Ophthalmol. 58, 409-414 (2014).

26. Hoffmann, P. C., Abraham, M., Hirnschall, N. \& Findl, O. Prediction of residual astigmatism after cataract surgery using swept source fourier domain optical coherence tomography. Curr. Eye Res. 39, 1178-1186 (2014).

27. Schröder, S. et al. Comparison of corneal tomography: repeatability, precision, misalignment, mean elevation, and mean pachymetry. Curr. Eye Res. 43, 709-716 (2018).

28. Oshika, T., Tomidokoro, A., Maruo, K., Tokunaga, T. \& Miyata, K. Quantitative evaluation of irregular astigmatism by Fourier series harmonic analysis of videokeratography data. Invest. Ophthalmol. Vis. Sci. 39, 705-709 (1998).

29. Kiuchi, G., Hiraoka, T., Ueno, Y., Mihashi, T. \& Oshika, T. Influence of refractive status and age on corneal higher-order aberration. Vis. Res. 181, 32-37 (2021).

30. Choi, S. K. \& Chang, J. W. Higher order aberration and astigmatism in children with hyperopic amblyopia. Korean J. Ophthalmol. 30, 53-59 (2016)

31. Cheng, X., Bradley, A., Hong, X. \& Thibos, L. N. Relationship between refractive error and monochromatic aberrations of the eye. Optom. Vis. Sci. 80, 43-49 (2003).

32. Leung, T. W., Lam, A. K. C. \& Kee, C. S. Ocular aberrations and corneal shape in adults with and without astigmatism. Optom. Vis. Sci. 92, 604-614 (2015).

33. Koch, D. D. et al. Contribution of posterior corneal astigmatism to total corneal astigmatism. J. Cataract. Refract. Surg. 38, 2080-2087 (2012).

34. Ueno, Y. et al. Age-related changes in anterior, posterior, and total corneal astigmatism. J. Refract. Surg. 30, 192-197 (2014).

35. Ueno, Y., Hiraoka, T., Miyazaki, M., Ito, M. \& Oshika, T. Corneal thickness profile and posterior corneal astigmatism in normal corneas. Ophthalmology 122, 1072-1078 (2015).

\section{Author contributions}

Y.U., T.H. and T.O. designed the study described here. Y.U, R.N., K.K, M.O. recruited participants and collected data. Y.U. and T.O. performed statistical analyses. T.O. drafted the main manuscript text and prepared figures. All authors reviewed and revised the manuscript prior to submission.

\section{Competing interests}

Dr. Tetsuro Oshika received compensation as a consultant from Alcon, Johnson \& Johnson Vision, Santen Pharmaceutical, and Mitsubishi Tanabe. He receives research support from Santen Pharmaceutical, Tomey, Alcon, Senju Pharmaceutical, Johnson \& Johnson Vision, HOYA, KOWA Pharmaceutical, Otsuka Pharmaceutical, Novartis, Pfizer, and Topcon.

\section{Additional information}

Correspondence and requests for materials should be addressed to T.O.

Reprints and permissions information is available at www.nature.com/reprints.

Publisher's note Springer Nature remains neutral with regard to jurisdictional claims in published maps and institutional affiliations.

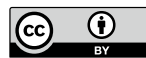

Open Access This article is licensed under a Creative Commons Attribution 4.0 International License, which permits use, sharing, adaptation, distribution and reproduction in any medium or format, as long as you give appropriate credit to the original author(s) and the source, provide a link to the Creative Commons licence, and indicate if changes were made. The images or other third party material in this article are included in the article's Creative Commons licence, unless indicated otherwise in a credit line to the material. If material is not included in the article's Creative Commons licence and your intended use is not permitted by statutory regulation or exceeds the permitted use, you will need to obtain permission directly from the copyright holder. To view a copy of this licence, visit http://creativecommons.org/licenses/by/4.0/.

(C) The Author(s) 2021 